\title{
Herramienta de Evaluación de Gestión para la Toma de Decisiones en la Fundación V\&C Basada en la NTC ISO 9001:2015.
}

\section{Assessment tool for decision-making management in the Fundación V\&C based on the NTC ISO 9001:2015.}

\author{
Jessica S. Hernández-Arteaga ${ }^{1}$, Lendy V. Rivera-Correa ${ }^{2}$, Oscar J. Cáceres-Rincón ${ }^{3}$, Juan C. Acevedo-Páez ${ }^{4}$ \\ ${ }^{1}$ Fundación V\&C, Cúcuta - Colombia, ${ }^{2,4}$ Universidad de Santander, Cúcuta - Colombia \\ ${ }^{3}$ Servicio Nacional de aprendizaje, Cúcuta - Colombia
}

Recibido: 13 de agosto de 2020.

Aprobado: 31 de octubre de 2020 .

\begin{abstract}
Resumen - El estudio de caso se desarrolla con el objetivo de mejorar la herramienta de evaluación actual para la toma de decisiones en la Fundación Valor y Compromiso (V\&C), con el apoyo de los líderes de proceso que aportaron información mediante la aplicacion de una encuesta, esta técnica permitió obtener un diagnóstico de la empresa en cuanto al uso de la herramienta actual. Seguidamente, se llevo a cabo la selección de los indicadores de gestión más significativos en cada proceso y el diseño de su respectiva ficha técnica. Luego, se consolidaron los resultados obtenidos del año 2019 en un instrumento de evaluación integral. En la siguiente etapa, se implementó una prueba piloto a los líderes de proceso, responsables del seguimiento y control de los indicadores de gestión, midiendo el desempeño de cada indicador a través de la calificación obtenida con relación a una meta proyectada. De esta forma, la herramienta de evaluación de gestión aporta al mejoramiento continuo de la organización, y se connota como un instrumento para la planeación y revisión de actividades. Asimismo, esta herramienta facilita la toma de decisión sobre las acciones preventivas y correctivas en los procesos que presentan un bajo o alto desempeño, mejorando los procesos más críticos como gestión comercial y gestión social, y manteniendo los procesos con mejor desempeño como jurídica y contratación y compras, según los resultados obtenidos en la prueba piloto realizada.
\end{abstract}

Palabras Claves: Herramienta de evaluación, Indicador de gestión, Medición del desempeño, Proceso, Toma de decisión.

\begin{abstract}
The case study is developed with the aim of improving the current evaluation tool for decision-making in the Fundación Valor y Compromiso (V\&C), with the support of process leaders who provided information through the application of a survey, this technique allowed to obtain a diagnosis of the company regarding the use of the current tool. Subsequently, the selection of the most significant management indicators in each process and the design of their respective technical sheet were carried out. Then, the results obtained for the year 2019 were consolidated into a comprehensive evaluation instrument. In the next stage, a pilot test was implemented for the process leaders, which are responsible for monitoring and controlling the management indicators, measuring the performance of each indicator through the qualification obtained concerning a projected goal. In this way, the assessment tool contributes to the continuous improvement of the organization, and connotes itself as an instrument for planning and reviewing activities. Likewise, this tool facilitates decision-making on preventive and corrective actions in processes that have low or high performance, improving the most critical processes such as commercial management and social management, and maintaining the best-performing processes such as legal, contracting and purchases, according to the results obtained in the pilot test carried out.
\end{abstract}

Keywords: Assessment tool, Management indicator, Performance measurement, Process, Decision-making. 


\section{INTRODUCCIÓN}

El sistema de gestión de la calidad, estipulado en la norma técnica colombiana [NTC] ISO 9001:2015 establece que la medición y el seguimiento permiten obtener información para la toma de decisiones y de acciones, así como para la mejora del desempeño institucional. Sin embargo, la norma no define una metodología de medición y evaluación para conocer los resultados bajo los principios de la calidad y su contribución a la toma de decisiones para la mejora continua [1].

Actualmente, la necesidad de realizar seguimiento y medición de los procesos, conlleva a que la organización deba construir una metodología que permita analizar el comportamiento de los diferentes procesos que participan en la transformación del producto o servicio [2]. Por ello se hace necesario identificar los objetivos de cada proceso para poder medirlo, analizarlo y mejorarlo. Asimismo, es importante llevar un registro de todas las mediciones que se obtengan en cada uno de los procesos que son sujeto de seguimiento, en pro de ajustar lo que se esté haciendo incorrectamente para mejorarlo [3]. Por tal motivo, en la investigación se abordó una metodología vinculada con la medición, análisis y evaluación de los diferentes procesos que conforman la Fundación V\&C, lineamientos establecidos en el numeral 9.1 de la NTC ISO 9001:2015, para alcanzar una mejora en el desempeño.

De otra parte, en la actualidad la calidad es una necesidad de todas las organizaciones, el avance exitoso de cualquier empresa va ligado a la posibilidad de satisfacer las necesidades y expectativas de sus clientes. Por tal motivo, se debe asegurar que la empresa tenga resultados medibles, que permitan analizar el estado actual de los objetivos establecidos con relación al proceso [4]. Por ello, el presente trabajo hace referencia a la mejora en la herramienta actual de evaluación de la Fundación V\&C, la cual tiene como función recolectar y procesar la información permitiendo el análisis de las actividades que se efectuan en cada proceso.

En la Fundacion V\&C existen problemas para el análisis en la medición de la gestión por parte de la alta direccion, debido a la ausencia de indicadores que puedan evaluar el desempeño real en su totalidad, monitorear el avance o cumplimiento adecuado de cada uno y retroalimentar los procesos, del mismo modo, afecta la toma de decisiones de forma oportuna sobre los proyectos que benefician a las comunidades, es por ello que el presente estudio quiera responder a la pregunta ¿Cómo se puede mejorar la herramienta actual que permita perfeccionar los indicadores de desempeño que aporten a la mejora continua de la organización?

Para dar respuesta a la problemática anterior, se plantea como objetivo de la investigacion proponer una mejora de la herramienta de evaluación de gestión actual para la toma de decisiones de la Fundacion V\&C. Se procede a realizar un estudio de caso para analizar el estado actual de la herramienta e identificar sus debilidades. Por otra parte, en conjunto con la alta dirección y los líderes de procesos se determinan las variables críticas y se establecen nuevos indicadores que suministran información para un cuadro integral que consolida los resultados de cada proceso y su estado de cumplimiento.

Finalmente, es de suma importancia la mejora en la herramienta de seguimiento, medición, análisis y evaluación ya que permite ordenar la información y medir los indicadores de gestión establecidos por la Fundación V\&C, por lo tanto se desarrollo un estudio de caso mediante parámetros que retroalimenten a la organización y permitan sustituir las apreciaciones subjetivas de los trabajadores por medidas objetivas al desempeño, cumplimiento de metas y obtención de resultados verídicos que contribuyan al análisis y a la mejora en la toma de decisiones para beneficio de la organización [5].

De otra parte, la Fundación V\&C es una organización sin ánimo de lucro que brinda apoyo a las Juntas de Accion Comunal (JAC) o a los asentamientos humanos organizados, zonas de alto riesgo, barrios no legalizados, es decir, desde la perspectiva de mercadeo la Fundación atiende a la base de la pirámide dentro de la sociedad [6], promoviendo y apoyando proyectos sociales, orientados a optimizar las condiciones en servicios públicos. La Fundación persigue fines de bienestar común, para una mejor calidad de vida de las comunidades ubicadas en sectores de alto riesgo en la ciudad de Cúcuta [7].

Desde la firma del contrato en mayo de 2011, se ha adquirido la experiencia que permite tener la suficiente ilustración sobre los aspectos relacionados con los beneficiarios y las dificultades para el mejoramiento de los servicios de acueducto y alcantarillado en las pilas públicas.

Interrelación de procesos. El sistema de gestión de la calidad de la Fundación V\&C, se definió y documentó de acuerdo con las normas internas y las adoptadas por la entidad, de la siguiente manera:

Identificación de procesos. Conocer los procesos que se desempeñan en la entidad y que tienen por labor el cumplimiento de los requisitos del cliente.

Agrupación de procesos. Luego de identificar y conocer los diferentes procesos, según el tipo de función que desempeñan dentro del sistema de gestión de la calidad se procede a agruparlos, logrando de esta forma el modelo P-H-V-A (Planear, Hacer, Verificar y Actuar) [8].

Proceso estratégico. Es el proceso encargado de analizar las necesidades y requerimientos de las partes interesadas, para garantizar la satisfacción de los usuarios o clientes de organización desde el componente estratégico [9]. Este proceso incluye los subprocesos de Direccionamiento Estratégico y Planeación del Sistema de Gestión.

Proceso misional. En este proceso se establece relación directa con el cliente (los procesos misionales requeridos para la realización del servicio, a partir de los cuales el cliente podrá percibir y valorar la calidad, comercialización y planificación del servicio) [9]. Este proceso está conformado por los subprocesos de Gestión Social y Gestión Comercial.

Proceso de soporte. En la organización, es el proceso responsable de proveer los recursos necesarios en su totalidad en cuanto a trabajadores, equipos e insumos, para otrogar el valor agregado esperado por los clientes [9]. Este proceso incluye los subprocesos de: Gestión Financiera, Gestión del Talento Humano, Contratación y Compras, Seguridad y Salud en el Trabajo y Jurídica.

En la figura 1 se observa el mapa de procesos de la Fundación $\mathrm{V} \& \mathrm{C}$ que demuestra la articulación de los procesos y subprocesos anteriormente descritos, con el objetivo de atender las necesidades y requerimientos de los clientes y partes interesadas.

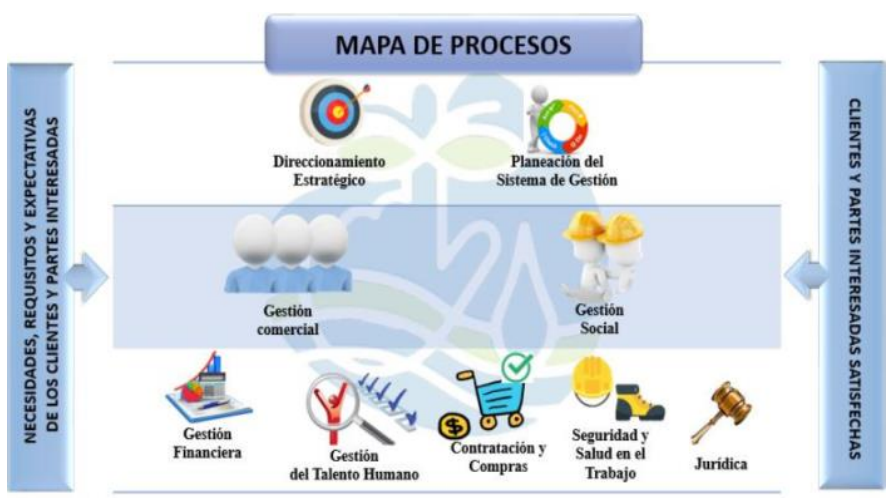

Figura 1: Mapa de procesos de la Fundación V\&C. Fuente: Tomado de [7]. 


\section{MARCO TEÓRICO}

Los indicadores son una expresión cualitativa o cuantitativa observable, utilizados para dar seguimiento y tomar decisiones en un sistema o proceso, con el fin de dar cumplimiento a los propósitos de una organización [10] [11]. Los indicadores son de gran ayuda para las empresas, definidos como Indicadores de gestión, ya que son una buena forma para asegurarse de llevar a cabo un control de las acciones y un buen análisis para conocer el cumplimiento o no de los objetivos propuestos, ya que aportan en la mejora de diferentes aspectos como, tomar decisiones correctas y oportunas, conocer la eficacia de las acciones, determinar puntos débiles de la empresa sobre los que se deben actuar para mejorarlos, identificar puntos fuertes para potenciarlos y conocer en tiempo real lo que sucede en la empresa para tomar medidas antes de que sea más difícil actuar sobre un problema [12] [13].

Los indicadores permiten evidenciar el nivel de cumplimiento de los objetivos organizacionales, a través de la medición de aspectos como recursos, cargas de trabajo, resultados de actividades, calidad de los productos y/o servicios, productividad y satisfacción del cliente [14] [15].

\section{a) Beneficios de los indicadores}

Contribuyen en la formulación de políticas de corto y largo plazo, y apoyan el proceso de planificación (i.e. establecer objetivos y metas) con mayor certeza y confiabilidad, a su vez optimizan el monitoreo de las tendencias en el entorno [16].

Permiten controlar y evidenciar la evolución de la compañía, de un departamento o de un área en particular [17].

Facilita la localización de procesos o áreas de la organización en las cuales existen inconvenientes de gestión como: uso inadecuado de los recursos, asignación del personal a las diferentes tareas, demoras desmedidas en la entrega de los productos, entre otros [18].

Promueven la satisfacción del cliente, como la determinación de las prioridades del cliente para alinear los objetivos institucionales, garantizando el logro de los resultados esperados gracias al monitoreo del grado de satisfacción del cliente a través de los indicadores [18].

Seguimiento del proceso, las mediciones son las herramientas básicas no sólo para encontrar las oportunidades de mejora, sino para aplicar las acciones. El mejoramiento continuo sólo es viable si se lleva a cabo un seguimiento exhaustivo a cada estapa de la cadena que constituye el proceso. [18].

Gerencia del cambio, un sistema de medición apropiado les permite a los trabajadores conocer cuáles son los resultados que sustenten la afirmación de que lo está realizando bien y su contribucion en las objetivos organizacionales [18].

Mejora la información respecto al uso y cumplimiento de recursos y actividades agregando valor al compromiso y la confianza de la entidad [19].

\section{b) Caracteristicas del indicador}

Hay tres características fundamentales que un indicador debe cumplir [20]:

Simplificación. La realidad en la que se actúa es multidimensional, un indicador no puede abarcar todas las dimesiones (económica, social, cultura, política) solo se puede considerar alguna.

Medición. Permite relacionar la situación actual de una dimensión de estudio respecto a patrones establecidos o modificados en el tiempo. Aporta al control y cumplimiento de la estrategia organizacional [21]

Comunicación. Para la toma de decisiones los indicadores deben transferir informacion.

\section{c) Tipologia de los indicadores}

La clasificación de los indicadores es muy variada y según la figura 2, se determinan seis o más categorías que la tipología de indicadores abarca, incluyendo a las dimensiones de evaluación de la economía, eficiencia, eficacia y calidad [22].

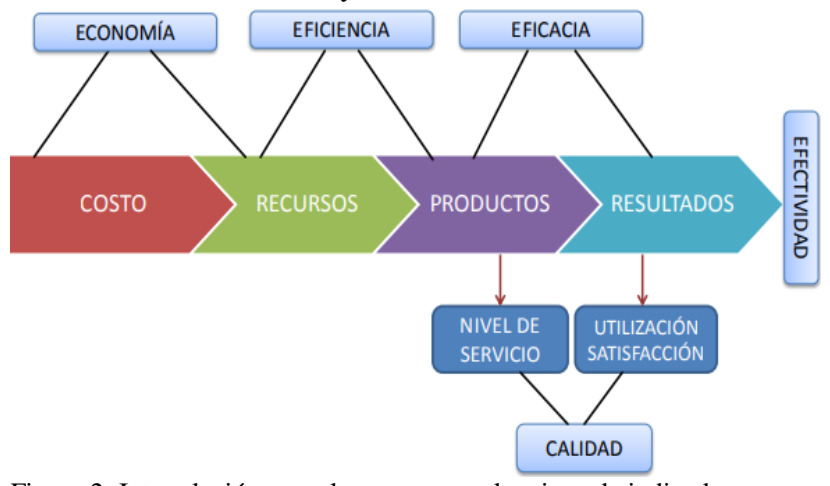

Figura 2: Interrelación entre los procesos y los tipos de indicadores.

Fuente: Tomado de [23].

Indicadores de eficacia. Son medidos por el grado de cumplimiento de los objetivos establecidos en sus actividades y planes de la entidad, o de los objetivos incorporados tácita o explícitamente en su misión. Es decir, comparar los resultados obtenidos con los planteados, independientemente de los medios utilizados [24].

Los indicadores de eficacia permiten identificar aspectos básicos del programa o la gestión que va a ser analizada mediante un sistema de información, ya que estos llevan de forma implícita la definición previa de objetivos y el seguimiento de éstos [24]. El grado de eficacia de estos indicadores se puede determinar mediante los siguientes criterios:

Contenido informativo. El indicador debe ser informativo, como un instrumento que posibilite la medición de una actividad, la identificación de logros, problemas y fallas que puedan estar afectando o beneficiando el rendimiento de los procesos. Debería entregarse información para la toma de decisiones que permita la mejora [25].

Formalidad. Un indicador de eficacia tiene que ser fiable para que consistentemente genere el mismo resultado cuando se utiliza de manera continua bajo las mismas condiciones [25].

Validez. El indicador debe ser válido, lo que significa que debe ser un instrumento adecuado de medición que garantice medir aquello que se requiere. Hay indicadores que arrojan resultados indirectos o estimaciones aproximadas y esto no significa que no sean válidos [25].

Apropiado. El indicador debe ser asignado al propósito para el cual se concibió. Es decir, las unidades y escalas deben ser las adecuadas, y las operaciones, las necesarias para implementar el análisis al resultado de acuerdo al proceso que se esté midiendo [25].

Factible. El indicador tiene que ser práctico, es decir, debe ser útil para medir aspectos concretos del servicio, de la actividad, de cualquiera de los procesos [25].

Indicadores de eficiencia. Se enfocan en el control de los recursos o las entradas del proceso. El análisis de la eficiencia se refiere a la evaluación efectuada en relación a los insumos adquiridos y su grado de aprovechamiento (entradas del proceso), que deben ser utilizados en la cantidad adecuada, en el menor tiempo, a un buen costo y con 
una excelente calidad. Por lo que se incorpora medios humanos, materiales y financieros [26].

Indicadores de efectividad. El análisis de este tipo de indicadores se refiere al logro de los resultados establecidos en el tiempo y con los precios más razonables posibles, involucrando asi a la eficiencia y la eficacia. Supone realizar lo adecuado con gran exactitud y sin desperdiciar tiempo o dinero [27].

Indicadores de calidad. Permiten evaluar las actividades de la organización que impactan directamente en el producto o servicio, y por tanto, en el cliente. Comprenden las siguientes subcategorías: oportunidad, pertinencia, compromiso, ética, crecimiento humano, utilidad, seguridad, adaptabilidad, manejabilidad, aceptación, estabilidad, consistencia y estética [16].

Indicadores de economía. Estos indicadores calculan la generación y gestión adecuada de los recursos financieros disponibles para alcanzar los objetivos, algunos indicadores típicos son, la capacidad de producción de recursos propios, cumplimiento presupuestal acorde con lo planificado y recuperación de cartera[23].

\section{d) Construcción de los indicadores}

En la figura 3 se muestran las actividades propuestas para construir un sistema de indicadores dentro de la organización, en total se definen siete etapas, las cuales consisten en definir la misión y objetivos, establecer la tipologia de indicadores, asignar responsabilidades, definir referentes, elaborar fórmulas, validar y comunicar los indicadores.

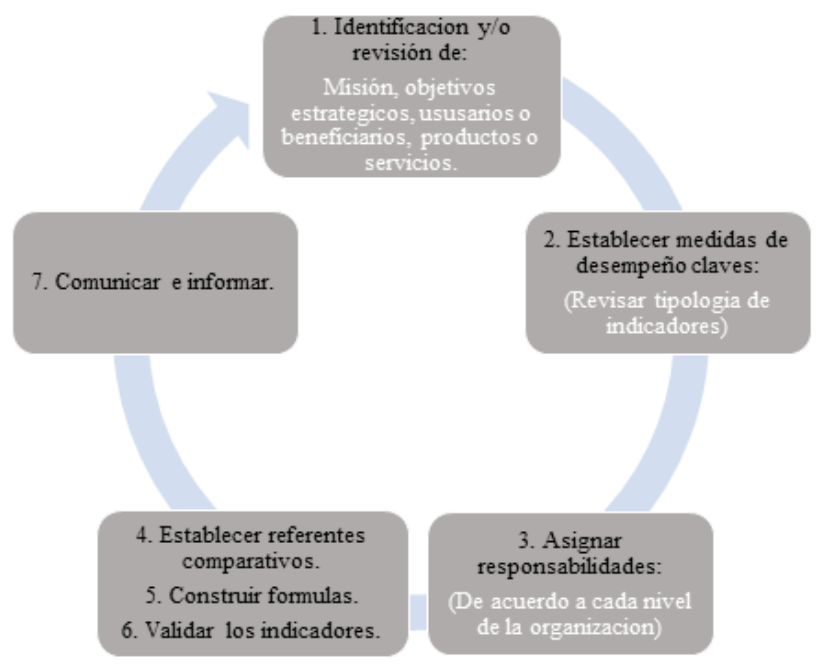

Figura 3: Etapas del proceso de construcción de indicadores.

Fuente: Adaptado de [18].

En la selección de indicadores se debe considerar un criterio de rentabilidad, dado que los recursos son siempre reducidos. A fin de encontrar los indicadores adecuados para el control y la toma de decisiones se deben considerar los siguientes criterios: i. la revisión y relación entre los indicadores y la misión, los objetivos estratégicos y los productos o servicios; ii. la revisión de la tipología de los indicadores estableciendo medidas de desempeño; iii. el cumplimiento de los procesos en el marco de la estrategia organizacional de acuerdo a la evolución de los factores criticos; iv. el establecimiento de referentes comparativos; v. la fiabilidad en el proceso del cálculo de los indicadores mediante la construcción de formulas y su respectiva validación; vi. la motivación del personal inducida por el indicador a ser comunicado; vii. la denominación del indicador debe responder a la mejor definición del objetivo para que no existan ambigüedades [28].

El calculo del indicador debe definir con veracidad las fuentes de información para recolectar los datos utilizados en el sistema, la periodicidad (e.g. mensual, trimestral, anual) con que se va a determinar el índice, el cual puede estar en forma de recuento, porcentaje, relación, cantidad [28].

Una vez construidos todos los indicadores de cada uno de los procesos que se desean controlar y analizar, se debe realizar la validación de los mismos. Para realizar una correcta validación se deben definir los criterios que se tendrán en cuenta para ello.

\section{METODOLOGÍA O PROCEDIMIENTO}

\section{a) Aspectos generales}

Para el desarrollo de la investigación se aplicó el método de estudio de caso, inicialmente se indentificaron los signos y síntomas del caso, luego se delimitó el aporte y el objeto del estudio, en la siguiente etapa se establecieron los procedimientos, sujetos y variables de estudio, finalmente se analizaron los resultados y se emitieron las inferencias y conclusiones de los investigadores del estudio desarrollado en la Fundación V\&C.

De acuerdo al nivel de la investigación, se adoptó un proceso descriptivo, según Hernández et al. "busca especificar propiedades, características y rasgos importantes de cualquier fenómeno que se analice" [29]. En esta investigación se analiza el estado de la herramienta de seguimiento, medición y evaluación, identificando las falencias y oportunidades de mejora para la Fundación V\&C. Adicionalmente la investigación se connota como cualitativa, de acuerdo a la naturaleza de la información, puesto que recurre a métodos por medio de "un proceso de recolección, análisis y vinculación de datos cualitativos en un estudio o una serie de investigaciones para responder al planteamiento del problema" [30].

Durante la investigación se implementaron instrumentos de recolección de información como cuestionarios, que permitieron un análisis estadístico de los resultados, logrando detectar las falencias presentes en la herramienta de medición de acuerdo a la percepción de los coordinadores de proceso. Además, se aplicó una lista de chequeo para la identificación de las variables críticas a medir por parte de la alta dirección y cada área de trabajo [31].

De otra parte, se llevó a cabo un muestreo intencional, donde se seleccionaron todos los elementos muestrales de la población y que aceptaron formar parte del estudio de caso [30]. La muestra objetivo del proyecto fue de ocho (8) personas, conformada por el Coordinador del sistema integrado de gestión, Coordinador de mantenimiento y reparaciones, Coordinador de cartera, Coordinador de proyectos, Coordinador de talento humano, Coordinador contable, Auxiliar administrativo y contable y Analista de Peticiones, Quejas y Reclamos (PQR), los cuales están capacitados para dar información y realizar aportes para la mejora de los procesos que lideran.

\section{b) Herramienta de evaluación actual}

Por medio de la herramienta se evalúa mensualmente lo planeado con los resultados obtenidos, mediante la medición de cada uno de los indicadores, verificando el cumplimiento de las metas y el logro del propósito organizacional. De este modo, la Fundación V\&C cuenta con un sistema de evaluación estándar que evalúa el rendimiento de los procesos de la organización, en un rango de 0 a 10 , fijando las metas en 6,66 .

Así mismo, para la calificación de cero (0) a diez (10) se trabaja en función de la proyección anual, el ejecutado (relación entre variables) y el rango de evaluación móvil. Cambiando si su tendencia es creciente o decreciente (ver figura 4), es decir, si con el indicador se busca maximizar o minimizar un resultado, por ejemplo, los ingresos económicos o accidentes laborales, respectivamente. 
Proyección anual (PA). Esta es la meta bajo la que cada proceso evalua su gestión a lo largo del año, no se puede modificar, a menos que sea dado por una decisión de junta de asamblea, es muy importante que este PA, el cual será equivalente al 6,66, se defina con mucha precisión basado en datos históricos y en comportamientos proyectados que tenga identificado el responsable del proceso.

Proyección mensual o modificada (PM). Presenta las mismas condiciones que el PA, es la meta de evaluación modificable que se convierte en PA al inicio de cada año de gestión, como su nombre lo indica se puede modificar siempre y cuando se conozca que la condición que afecta negativa o positivamente se mantendrá en años futuros (bajo previa autorización de Gerencia), ya sea producto de una tendencia por meses o alguna condición especial en cualquier año futuro, que pueda ser prevista desde la actualidad. En el caso de evidenciar que algo impactará los indicadores, pero no afectará años futuros no se modifica el PM, sin embargo, se debe tener muy en cuenta esta razón a la hora de realizar el análisis de las calificaciones.

Ejecutado $(\boldsymbol{E})$. Es la relación entre las variables del indicador, muestra el comportamiento obtenido y que se debe dar de carácter mensual y anualizado (siendo este el conglomerado del año hasta la fecha en curso) se califica versus el PA.

Rango de evaluación móvil (REM). Este rango de evaluación define los límites mínimos y máximos de la calificación que puede obtener un indicador. Entre más amplio sea el REM mayor dificultad se tendrá en obtener el (10) o el (0). Su definición es definida por cada responsable del proceso y se debe ajustar pensando en el comportamiento histórico del indicador.

Calificación de indicadores (CI). Para los indicadores de gestión la calificación que se da entre 0 y 10 ; se calcula mediante la fórmula de la pendiente, con una variación que es la tendencia del indicador, esto se debe a que no todos tienen el mismo comportamiento.

En forma general, se establece que para los indicadores de gestión con tendencia creciente $(+)$, la mínima calificación es obtenida cuando se logre como resultado en el índice un valor igual a la proyección anual con diferencia de dos REM en sentido contrario, y la máxima calificación se obtendrá cuando se logre como resultado en el índice un valor igual a la proyección anual con un REM a favor; siendo así los 2/3 del rango de calificación los correspondientes al logro de la meta como resultado del índice (ver ecuación 1).

Asimismo, para los indicadores de gestión con tendencia decreciente (-), se establece que la máxima calificación es obtenida cuando se logre como resultado en el índice un valor igual a la proyección anual con diferencia de un REM en sentido contrario, y la mínima calificación se obtendrá cuando se logre como resultado en el índice un valor igual a la proyección anual con dos REM a favor, siendo así 1/3 del rango de calificación los correspondientes al logro de la meta como resultado del índice (ver ecuación 2).

Para indicadores con tendencia creciente $(+)$ se define la siguiente fórmula:

$$
\begin{array}{r}
\mathrm{CI}=\frac{(10 \times(2 \mathrm{REM}+\mathrm{Z}-\mathrm{PA}))}{(3 \mathrm{REM})} \\
C I_{\text {máx }}=\mathrm{PA}+\mathrm{REM} \\
C I_{\text {min }}=\mathrm{PA}-2 \mathrm{REM}
\end{array}
$$

Para indicadores con tendencia decreciente (-) se define la siguiente fórmula:

$$
\begin{gathered}
\mathrm{CI}=\frac{(10 \times(2 \mathrm{REM}-\mathrm{Z}+\mathrm{PA}))}{(3 \mathrm{REM})} \\
C I_{\text {máx }}=\mathrm{PA}-\mathrm{REM}
\end{gathered}
$$

$$
C I_{\text {min }}=\mathrm{PA}+2 \mathrm{REM}
$$

Donde $Z$ corresponde al valor del factor evaluado del indicador que se haya definido.

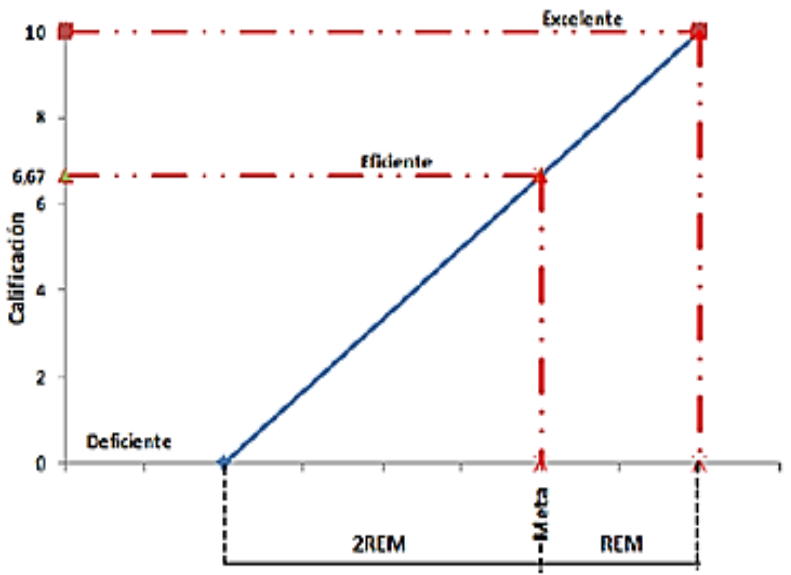

(a)

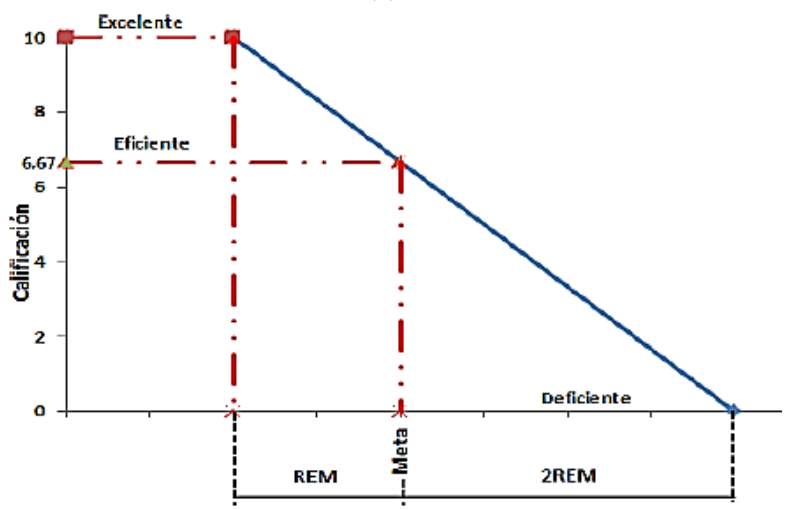

(b)

Figura 4: Tendencia de los indicadores, (a) creciente, (b) decreciente. Fuente: Tomado de [32].

Cada indicador consta de una nota para el período y otra para el desempeño acumulado; donde la nota del período evalúa los resultados del mes, mientra que la nota acumulada mide la tendencia de los resultados.

\section{RESULTADOS, ANÁLISIS E INTERPRETACIÓN}

En los resultados obtenidos en el diagnóstico, se evidencia que la mayoría de las áreas evaluadas adoptan los indicadores de gestión establecidos, no obstante, existe una apatía para la implementación de estos como herramienta de medición, análisis y evaluación de las actividades realizadas. Una vez aplicado el cuestionario a los líderes de proceso, se continuó con la representación estadística, en la figura 5 se muestra el resumen de los resultados obtenidos de las preguntas con mayor relevancia. Se puede observar que el proceso de Gestión Social es el más crítico, puesto que no cuenta con indicadores en la herramienta actual que le permite tomar decisiones objetivas y basadas en datos precisos. De igual forma, se destaca la inconformidad de todos los líderes, debido a que no cuentan con la opción de realizar un análisis de resultados por medio de una representación grafica. Adicionalemente, la alta gerencia manifestó la gran necesidad de mejoras en la herramienta y la creación de indicadores adecuados en los procesos carentes de ellos. Por otra parte, se identificó que los procesos de gestión financiera, jurídica y seguridad y salud en el trabajo se encuentran conformes con el uso de la herramienta y sus indicadores. 
¿Considera usted que el indicador se comunica efectivamente?

¿La periodicidad establecida para el análisis del indicador es adecuada?

¿La representación gráfica utilizada para analizar el comportamiento del indicador es comprensible?

¿Considera usted que los indicadores de gestión son apropiados para la toma de decisiones?

¿Los indicadores de gestión definidos en su proceso son útiles?

¿Usted ha participado en la construcción de los indicadores de gestión?

\author{
घ Gestión Comercial (Cartera) \\ ఐ Planeación del Sistema de Gestión \\ - Gestión Social \\ a Contratación y compras \\ - Juridica
}

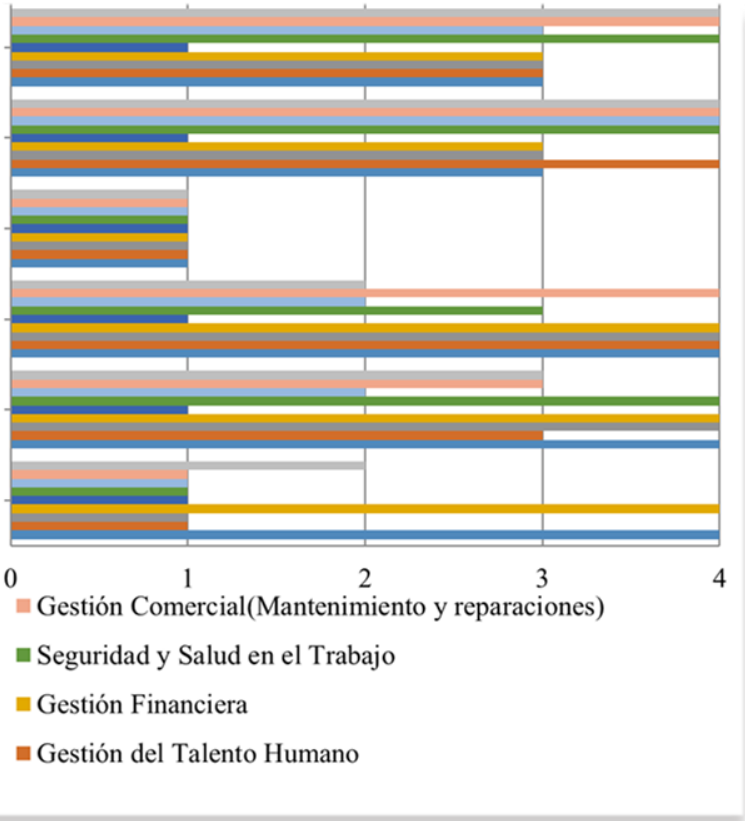

\section{b) Determinación de las variables críticas de medición y los indicadores claves}

Figura 5: Diagnóstico aplicado a líderes de proceso.

Fuente: Elaboración propia.

\section{a) Identificación de causas}

Una vez elaborado el diagnóstico de la herramienta actual, se identifcaron las principales causas y sub-causas que ocasionan la deficiencia en el análisis de la información y la toma de decisiones de los procesos de la Fundación V\&C (i.e. Efecto o Problema), dichas falencias se representan en un diagrama de causa-efecto o Ishikawa [33], [34] (Ver figura 6).

Las categorías que se tomaron como soporte para identificar las mejoras de la herramienta actual fueron procesos y método, donde se identificaron causas como deficiencia en la herramienta empleada, ausencia de control de información más detallada, desconocimiento de metodologías en el proceso de medición debido a la apatía en la implementación de indicadores, desconocimiento de la formulación y procedimiento de la estrategia de proyecto por parte de los coordinadores de proceso, así como las falencias en la estructura de los indicadores de gestión.
Para la identificación de las variables críticas a medir en los procesos de gestión de la Fundación V\&C, se diseñó un cuestionario aplicado a la gerencia con el fin de priorizar los procesos que requieren mejoras, concluyendo que el proceso de Gestión Comercial es uno de los más críticos, ya que este proceso permite llevar a cabo un análisis de los planes estratégicos que se deben plantear para cada una de las comunidades; el segundo proceso critico es Gestión Social, puesto que no cuenta con variables de medición que apoyen la toma de decisiones en temas relevantes para la Fundación V\&C como el desempeño de proyectos.

De igual manera, la gerencia considera que la interpretación gráfica de los resultados sería de gran ayuda para visualizar el rendimiento del proceso y desarrollar un análisis más rápido y eficaz.

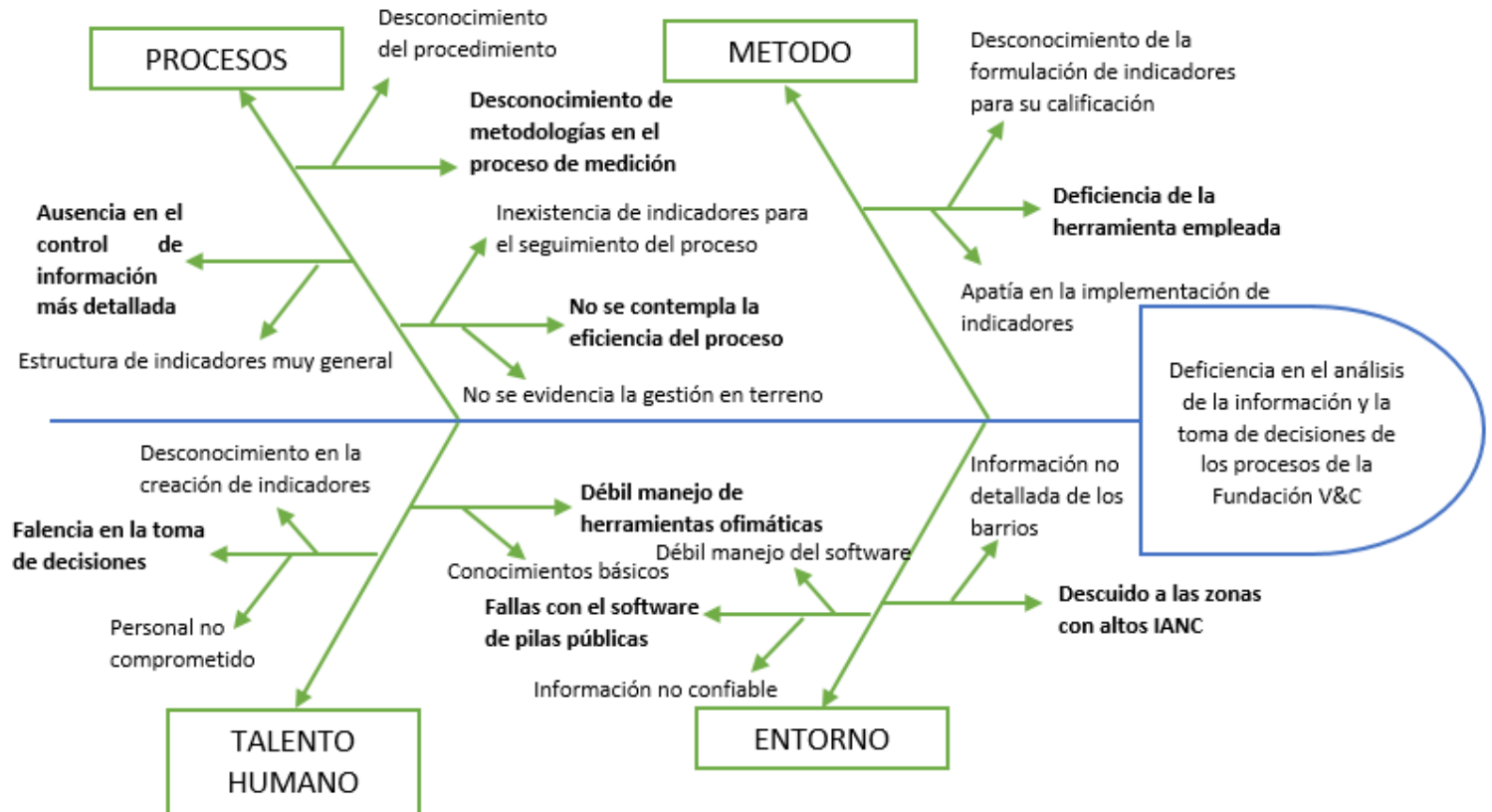

Figura 6: Diagrama Causa-Efecto.

Fuente: Elaboración propia. 
Para la definición del porcentaje de incidencia de los indicadores de gestión de la Entidad, se tomó como base una lista de chequeo de los indicadores inherentes a cada uno de los procesos de la Fundacion $\mathrm{V} \& \mathrm{C}$, los cuales son consolidados para desarrollar los informes de junta directiva. Mediante un grupo focal donde participaron los líderes de cada área, se identificaron los indicadores de mayor importancia en cada uno de los procesos referidos en la tabla 1, definido como el porcentaje de incidencia actual [35].

Tabla 1: Lista de chequeo de indicadores.

\begin{tabular}{|l|l|l|}
\hline Proceso & Indicador & $\begin{array}{c}\text { \% Incidencia } \\
\text { Actual }\end{array}$ \\
\hline $\begin{array}{l}\text { Planeacion del Sistema } \\
\text { de Gestión }\end{array}$ & $\begin{array}{l}\text { Autogeneracion de } \\
\text { ACPM's* }\end{array}$ & $40 \%$ \\
\hline \multirow{2}{*}{\begin{tabular}{l} 
Gestión Comercial \\
\cline { 2 - 3 }
\end{tabular}} & $\begin{array}{l}\text { Porcentaje de agua } \\
\text { consentida }\end{array}$ & $10 \%$ \\
\cline { 2 - 3 } & Número de quejas & $20 \%$ \\
\hline Gestión Social & Ninguno & $15 \%$ \\
\hline Gestión Financiera & Efectividad en costos & $100 \%$ \\
\hline $\begin{array}{l}\text { Contratacion y } \\
\text { Compras }\end{array}$ & Seguimiento a proveedores & $40 \%$ \\
\hline Seguridad y Salud & Accidente de trabajo & $17 \%$ \\
\hline $\begin{array}{l}\text { Gestión de Talento } \\
\text { Humano }\end{array}$ & $\begin{array}{l}\text { Ausentismo por hora } \\
\text { trabajador }\end{array}$ & $30 \%$ \\
\hline \multirow{2}{*}{ Juridica } & $\begin{array}{l}\text { Defensa oportuna acciones } \\
\text { de tutela }\end{array}$ & $60 \%$ \\
\hline
\end{tabular}

Fuente: Elaboración propia. mejora

*ACPM's: Autogeneración de acciones correctivas, preventivas y de

\section{c) Diseño de la herramienta}

La mejora de la herramienta de medición, análisis y evaluación, está desarrollada a través de una interfaz de fácil manejo y dominio para los coordinadores de procesos por medio del programa informático Microsoft Excel $^{\circledR}$, la cual se diligencia de acuerdo a la frecuencia del indicador. Durante el diseño de la herramienta se toman en cuenta todos los requerimientos realizados por cada una de las partes interesadas, logrando evidenciar en ella diferentes características que aportan a la descripción del indicador [36], tales como: proyección mensual (PM), proyección anual (PA), REM, histórico mensual, calificaciones, tendencia y comportamiento del indicador, igualmente el análisis de los datos obtenidos con la formulación del plan acción si es necesario.

Como resultado, se reflejan cambios en la estructura del diseño de la herramienta de medición, análisis y evaluación, la cual contiene un conjunto de fichas técnicas que permite obtener información acerca del comportamiento del indicador más importante, seleccionado anteriormente por cada uno de los líderes de proceso. La herramienta está dividida en varios componentes en una matriz en Microsoft Excel $^{\circledR}$, en primera instancia cuenta con la página de inicio, que contiene el mapa de procesos de la Fundacion V\&C, donde se selecciona el proceso a analizar y a su vez lo direcciona a las fichas técnicas de indicadores, generando una navegación más práctica dentro de la herramienta [5]. Seguidamente en las fichas técnicas de cada indicador de gestión, cada ficha posee una estructura de 6 ítems que permite obtener la mayor información sobre el indicador de interés (ver figura 7) [37], [38], a continuación, se describe cada ítem.

Objetivo. Se refiere al objetivo del proceso que se esta evaluando, que forma parte del mapa de procesos.

Definición del indicador. Se da a conocer el nombre, objetivo, unidad de medida, frecuencia, incidencia, tendencia y el responsable de la medición del indicador.

Información para la medición del indicador. Se describe la fuente de información y la fórmula de cálculo del indicador, también se especifica la proyección anual (PA), la proyección mensual (PM), el rango de evaluación de meta (REM). Además, se calcula la calificación simbolizada con un color propio, verde, amarillo y rojo, esto permite determinar el estado del indicador según los tres rangos establecidos como óptimo, aceptable y deficiente, respectivamente, facilitando el análisis de las variaciones e implementar acciones correctivas [5], [39].

Comportamiento del indicador. Esta sección de la ficha muestra los resultados de cada mes, de las variables descritas en la fórmula de cálculo del indicador.

Medición. Se procede a realizar los cálculos para comprender el comportamiento del indicador a lo largo de cada mes, lo anterior se obtiene del desarrollo de las variables, calificación del periodo y calificación acumulada, luego se podrá observar la gráfica que facilita la interpretación de los resultados.

Análisis de los resultados. Después de obtener los resultados del comportamiento del indicador, se procede a realizar el respectivo análisis teniendo en cuenta el periodo evaluado y el periodo siguiente. Con base en lo anterior, se determina si el indicador amerita acción o no, y con ello formular un plan de acción en el que se describa la solicitud de acción correctiva (SAC), la solicitud de acción preventiva (SAP) o la solicitud de acción de mejora (SAM).

Descripción. Finalizando la ficha se encuentra una breve descripción del indicador, donde se refiere información como la incidencia, calificación y la nota del periodo y acumulado.

Evaluación de desempeño. Por último se efectúa un análisis de la ficha técnica, donde se ejecuta una evaluación de desempeño al indicador de acuerdo a lo establecido en el literal 9.1 de la NTC ISO 9001:2015 [40], identificando las falencias o virtudes presentadas en cada periodo. 


\section{FICHA TECNICA DE INDICADORES DE GESTIÓN}

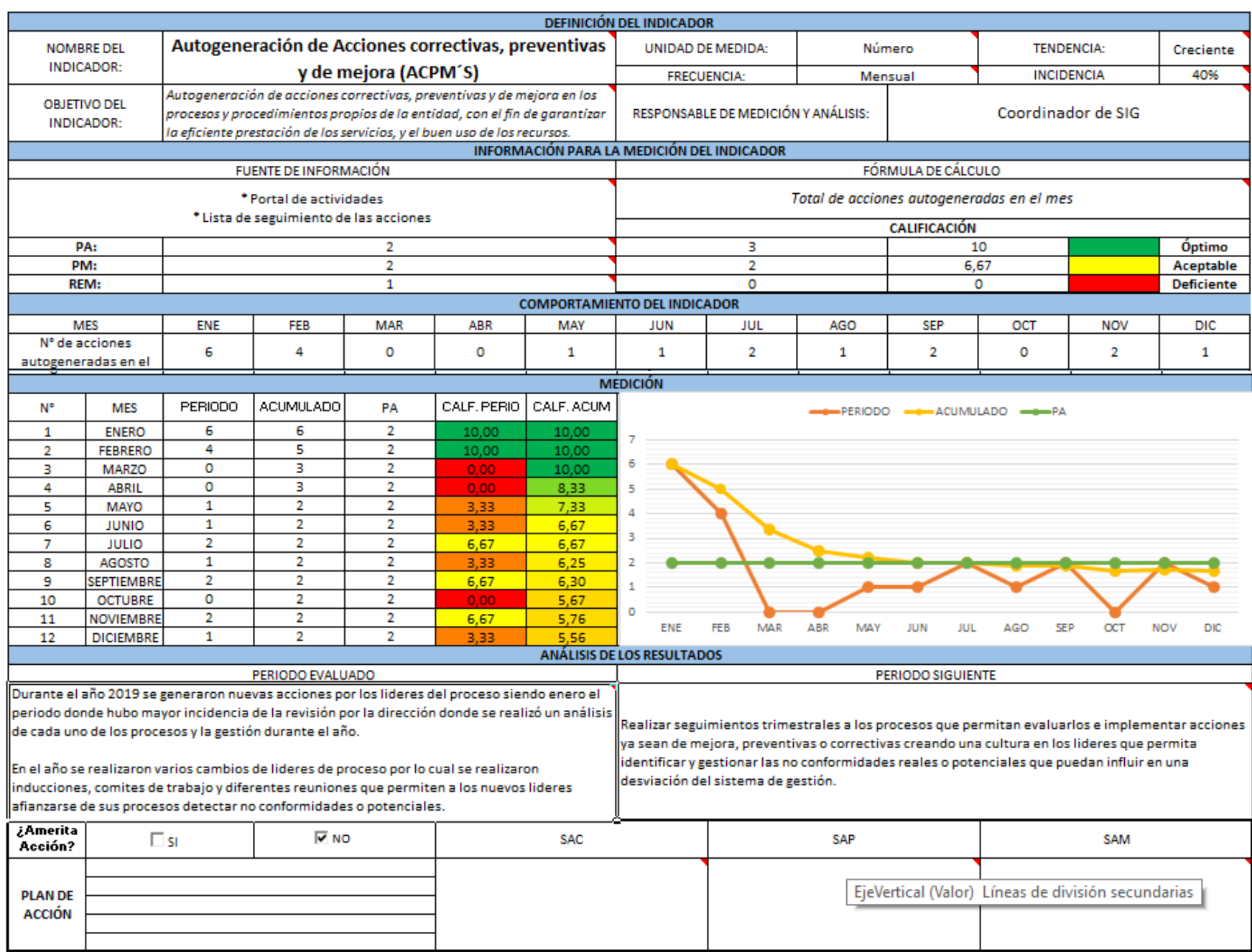

\begin{tabular}{|c|c|c|c|c|}
\hline DESCRIPCION & INCIDENCI & \multicolumn{2}{|c|}{ NOTA } & \multirow{2}{*}{ CALIF } \\
\cline { 3 - 4 } & A & PERIODO & ACUMULADO & \\
\hline $\begin{array}{l}\text { Autogeneración de Acciones correctivas, } \\
\text { preventivas y de mejora (ACPM'S) }\end{array}$ & $40 \%$ & 10,00 & $\mathbf{1 0 , 0 0}$ & 4,00 \\
\hline & $40 \%$ & $\mathbf{4 , 0 0}$ & $\mathbf{4 , 0 0}$ & $\mathbf{4 , 0 0}$ \\
\hline
\end{tabular}

Figura 7. Ficha tecnica de indicador de gestión.

Fuente: Elaboración propia.

\section{PROPUESTA DE SOLUCIÓN O MEJORAS}

Al finalizar el diseño de la herramienta de seguimiento, análisis y evaluación se efectuó una prueba piloto a través de los líderes de área, indicando inicialmente el manejo adecuado de la nueva herramienta, así mismo se hizo entrega de la misma para que cada uno pudiese implementarla agregando datos del periodo 2019 para validar su funcionamiento (ver figura 8 ).

Según se muestra en la figura 7, el indicador autogeneración de acciones correctivas, preventivas y de mejora correspondiente al proceso de planeación del sistema de gestion, presentó el siguiente análisis, durante el periodo evaluado se generaron acciones por el líder del proceso, siendo enero y febrero los meses donde hubo mayor incidencia en la revisión por la dirección, por otra parte durante el mes de marzo y abril hubo un cambio de personal en esta área lo cual causó un resultado deficiente. El líder a cargo planteó para el siguiente período realizar seguimiento a todos los procesos por medio de evaluaciones y así implementar las respectivas acciones.

En la parte inferior de cada ficha técnica, los líderes de procesos dieron a conocer la perspectiva sobre la nueva herramienta, donde manifestaron el agrado y el buen desempeño de la mejora realizada, resaltaron el orden y la facilidad para agregar los datos, asi mismo, dieron valor apreciativo a la gráfica incluida en cada ficha, puesto que ofrece una visualización rápida sobre el comportamiento de los indicadores, aportando a la obtención de información explícita para el análisis y evaluación, que conducen de manera oportuna a la toma de decisiones. 

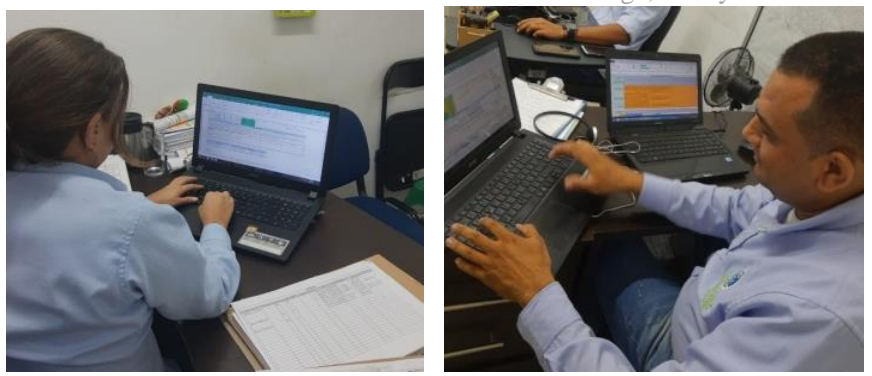

Figura 8: Registro fotográfico de prueba piloto.

Fuente: Elaboración propia.

Los líderes de los procesos realizaron un análisis de los resultados obtenidos en la prueba piloto con los datos del período 2019, en cada ficha técnica se definieron las acciones a seguir en el siguiente periodo.

Planeación del sistema de gestión. En el periodo 2019 se generaron nuevas acciones por los líderes de proceso, siendo enero el mes donde hubo mayor incidencia en la revisión por la dirección realizándose un análisis de la gestión durante el año.

Gestión financiera. En el período evaluado se mantuvo un buen promedio en los gastos relacionados con el presupuesto, sin embargo, hubo un incremento en los meses de febrero y septiembre por el ingreso de nuevo personal, que no permitió cumplir con lo presupuestado.

Gestión de talento humano. Se realizó el respectivo seguimiento y la generación de permisos y registro de incapacidades; durante el mes de abril se presentaron varios eventos por enfermedad general que ocasionó la ausencia de varios de los trabajadores, por cual para los siguientes meses se les otorgaron a cada uno de los lideres un número de permisos que podrían asignar por mes con el fin de reducir el número de horas perdidas.

Contratación y compras. Se realizaron compras y servicios a proveedores existentes y nuevos, a cada uno se les aplicó la evaluación inicial y de seguimiento, obteniendo calificaciones bajas para algunos, conllevando a la no compra o contratación de servicios por motivo de insatisfacción.

Seguridad y salud en el trabajo. Se registraron varios eventos por accidente de trabajo que generaron el incumplimiento del indicador durante varios meses, para ello se realizaron los diferentes informes a cada uno de los casos y su respectiva investigación.

Jurídica. En el periodo analizado se logró dar respuesta oportuna a las acciones de tutela por parte de los accionantes, con un tiempo promedio de respuesta de un día hábil, lo cual evidencia la eficacia en el proceso y cumplimiento de las metas trazadas, las cuales correspondieron a fallas de prestación del servicio por parte del operador.

Gestión comercial. El recaudo obtuvo un promedio de $\$ 137.000 .000$ de pesos. La gestión realizada se ve reflejada con un mayor impacto en los meses de enero, marzo, mayo y noviembre. Se realizaron jornadas de normalización de cartera en terreno, cortes a beneficiarios con más de tres meses de atraso y vinculación de beneficiarios nuevos que realizaron aportes. Se realizó flexibilización de las condiciones de financiación para lograr que los beneficiarios con menos capacidad adquisitiva puedan normalizar sus deudas.

Por otra parte, durante el mismo periodo hubo un promedio de 54\% de agua consentida, los meses de mayor desperdicio de agua fueron mayo, julio y noviembre. Para reducir este indicador se tomaron en cuenta varias acciones como, mejorar la comunicación con las juntas de accion comunal para poder realizar reparaciones de tuberia de manera oportuna, actulización de censo para vincular a los beneficiarios que no estuvieran realizando sus pagos a la deuda de
Aguas Kpital, realizar campañas de utilizacion del agua en los barrios y efectuar cortes de mangueras y conexiones irregulares del sistema de tuberias.

También hubo un alto número de quejas, cuyo motivo fueron las fallas en el servicio de agua, ya que hubo meses en los que por la turbidez del agua fue necesario suspender el servicio, la ampliación de la planta del Pórtico redujo la capacidad de producción del recurso hídrico potable viendo afectadas las zonas de alto riesgo. Sin embargo, se mantuvo comunicación constante con la comunidad y los presidentes de las Juntas de Acción Comunal para llevar carrotanques con agua potable a las zonas afectadas.

Gestión social. En el cumplimiento de plan de obras de la comunidad en el 2019, se destaca que, si la comunidad no ha pagado el $70 \%$ del costo que les corresponde por proyecto, no se da inicio a la obra. Esta es la razón por la cual, muchas veces no se ejecutan las obras como se planean en el periodo.

En la tabla 2 se muestran los resultados obtenidos de la prueba piloto realizada en el mes de enero del 2020 consolidados en el informe de gestión de la herramienta de seguimiento, análisis y evaluación. En la prueba no se tuvieron en cuenta la totalidad de los indicadores que comprenden los procesos. Por otra parte, se puede observar que los procesos con mayor desempeño fueron Gestión Financiera, Contratación y Compras, Seguridad y Salud en el Trabajo y Jurídica, en virtud de que estas áreas dieron cumplimiento a las actividades programadas. 
Tabla 2. Informe de gestión de la entidad Fundacion V\&C

\begin{tabular}{|c|c|c|c|c|c|}
\hline \multirow{2}{*}{ Proceso } & \multirow{2}{*}{ Objetivo } & \multirow{2}{*}{ Incidencia } & \multicolumn{2}{|l|}{ Nota } & \multirow{2}{*}{ Calif } \\
\hline & & & Período & Acumulado & \\
\hline $\begin{array}{l}\text { Planeación del } \\
\text { Sistema de Gestión }\end{array}$ & $\begin{array}{l}\text { Establecer el direccionamiento estratégico de la Entidad así como la Política } \\
\text { que garanticen el enfoque al cliente de la organización y su comunicación y } \\
\text { cumplimiento a través del Sistema de Gestión en todos los niveles de la } \\
\text { organización. }\end{array}$ & $7 \%$ & 4,00 & 4,00 & 0,3 \\
\hline Gestión Financiera & $\begin{array}{l}\text { Administrar y consolidar la información financiera y tributaria de la } \\
\text { Fundacion V\&C y de sus actividades, enmarcados dentro de los procesos } \\
\text { contables, presupuestales y de tesorería. }\end{array}$ & $8 \%$ & 8,72 & 8,72 & 0,7 \\
\hline $\begin{array}{l}\text { Gestión del Talento } \\
\text { Humano }\end{array}$ & $\begin{array}{l}\text { Desarrollar integralmente el talento humano vinculado a la fundación, en pro } \\
\text { del mejoramiento continuo, la satisfacción del personal y el desarrollo } \\
\text { organizacional que permita contar con el personal idóneo y competente para } \\
\text { lograr con los objetivos planteados. }\end{array}$ & $15 \%$ & 2,62 & 2,62 & 0,4 \\
\hline $\begin{array}{l}\text { Contratación y } \\
\text { Compra }\end{array}$ & $\begin{array}{l}\text { Gestión en la adquisición de bienes y servicios necesarios para la operación } \\
\text { de la fundación, con las mejores condiciones del mercado (precio, calidad, } \\
\text { tiempo de entrega). }\end{array}$ & $7 \%$ & 6,67 & 6,67 & 0,5 \\
\hline $\begin{array}{l}\text { Seguridad y Salud en } \\
\text { el Trabajo }\end{array}$ & $\begin{array}{l}\text { Procurar el cuidado integral de los trabajadores y mantener en óptimas } \\
\text { condiciones sus instalaciones, herramientas y demás objetos que utilizan en } \\
\text { su labor diaria. }\end{array}$ & $15 \%$ & 10,00 & 10,00 & 1,5 \\
\hline Jurídica & $\begin{array}{l}\text { Orientar, asesorar y defender la fundación V\&C en asuntos jurídico- } \\
\text { administrativos internos y externos de su competencia, velando de manera } \\
\text { oportuna y eficaz por los intereses de la misma. }\end{array}$ & $8 \%$ & 10,00 & 10,00 & 0,8 \\
\hline Gestión Comercial & $\begin{array}{l}\text { Mantener actualizada la base de datos de los beneficiarios de las pilas públicas } \\
\text { de forma precisa, confiable y oportuna; logrando los mayores índices de } \\
\text { recaudo y la satisfacción del beneficiario. }\end{array}$ & $20 \%$ & 4,08 & 4,08 & 0,8 \\
\hline Gestión Social & $\begin{array}{l}\text { Contribuir con el desarrollo sostenible y organizado de las comunidades } \\
\text { vinculadas al plan conéctate de manera oportuna, precisa y eficiente; } \\
\text { buscando el progreso, desarrollo y bienestar de las comunidades y la } \\
\text { concientización de sus compromisos con la fundación. }\end{array}$ & $20 \%$ & 2,91 & 2,91 & 0,6 \\
\hline
\end{tabular}

Fuente: Elaboración propia.

\section{CONCLUSIONES}

En el caso estudiado se identificaron como signos principales que la herramienta actual de seguimiento, medición y análisis, presenta un débil manejo de la misma y la ausencia de medición para la aplicación de controles en los procesos de la Fundación V\&C, en el análisis de los resultados se evidenció que dichas falencias se deben en gran parte a la poca participación de los líderes de área en el diseño e implementación de los indicadores y el poco conocimiento de la estructura de los mismos. Lo anterior ha generado que desde la Alta gerencia no exista un método eficaz para la toma decisiones de forma oportuna y basada en información veraz, conllevando a afectaciones en el normal desarrollo de proyectos en la comunidad.

El listado maestro permitió consolidar los indicadores de toda la organización y por medio de una selección realizada por los líderes de proceso, se definieron los más importantes y aquellos que se debían mejorar o diseñar para el caso de los procesos que no contaban con ellos (e.g. Gestión Social). Desde la perspectiva de la gerencia, los procesos más críticos dentro de la empresa son Gestión Social y Gestión Comercial, por ello, se determinó diseñar la herramienta incluyendo cada uno de los procesos, definiendo los porcentajes de acuerdo al grado de importancia del proceso que permitieron la evaluación del desempeño, los cuales fueron, para Gestión Comercial y Social el 20\%, Seguridad y Salud en el Trabajo y Gestión del Talento Humano el 15\%, Gestión Financiera y Jurídica el 8\%, finalmente Planeación del Sistema de Gestión y Contratación y Compras con el $7 \%$.

En el contexto organizacional, la nueva herramienta de seguimiento, medición y análisis para la Fundación V\&C, ha sido exitosa, debido al impacto positivo reflejado por los líderes de proceso al final de cada ficha técnica, en virtud del cumplimiento de los requisitos sugeridos por la gerencia y la mejora de las falencias referidas por los líderes. Finalmente, la herramienta diseñada para la toma de decisiones en los diferentes procesos de la entidad, ha mejorado significativamente en profundidad y contenido la herramienta anterior, además ha permitido la participación activa y el aporte de los líderes del proceso lo cual conlleva a una implementación efectiva de la misma.

\section{RECOMENDACIONES}

Proceder a la implementación de la herramienta de seguimiento, medición y análisis con cada uno de los indicadores existentes; direccionados a los objetivos estratégicos que permita el cumplimiento de los mismos y de esta manera aumentar la eficacia en cada uno de los procesos.

Actualizar los documentos y registros existentes en el sistema de gestión de la calidad, de acuerdo a cambios presentados en el proceso de implementación de la herramienta de seguimiento, medición y análisis. De igual forma, dar a conocer la medición y análisis de los indicadores al personal del proceso y al gerente general, con el fin de establecer acciones preventivas y/o correctivas en las situaciones de incumplimiento de la proyección anual establecida.

Desarrollar programas de capacitación y sensibilización a los coordinadores de proceso, a fin de crear un hábito interno en el manejo adecuado y oportuno de las herramientas implementadas por la organización, en pro de una cultura interna basada en la importancia del sistema de gestión de la calidad, logrando con ello la obtención de los objetivos planteados por la organización. Tener en cuenta otras metodologías para la toma de decisiones a nivel empresarial, las cuales no se consideraron en esta investigación, como el cuadro de mando integral, la matriz de decisión, el modelo de toma de decisiones cuantitativo, entre otras. 


\section{REFERENCIAS}

[1] J. Crespo, "Metodología de medición y evaluación -MESGCpara seguimiento, análisis y mejora de los sistemas de gestión de calidad," vol. 7, nº 1, pp. 107-123, 2015.

[2] I.Y. Esguerra, L.G. Lozano, L.D. Villamizar y J.C. Acevedo, "Metodologia Para la Estandarización del Modelo Normativo en Centrales Electricas de Norte de Santander - Grupo EPM," Revista de investigacion en administracion y energia, vol. IV, $\mathrm{n}^{\circ}$ 2, 112016.

[3] Centro Europeo de Postgrados, "SGC en la Empresa: Seguimiento, Medición, Análisis y Evaluación,". [En línea]. Disponible en: https://www.ceupe.com/blog/sgc-empresaseguimiento-medicion-analisis.html.

[4] D.M. Alfonso-Ortiz y L.C. Soto-Ramirez, "Propuesta de plan de mejora para el proceso de Soporte al Cliente del área Comercial de Kantar IBOPE Media Colombia S.A.S., con base en los lineamientos del numeral 9.1 de la NTC-ISO 9001-2015", tesis Especialización, Uni. Libre, 2017.

[5] L.S. Resterpo-de-Ocampo, S. Estrada-Mejía y C. RamirezAristizabal, "Modelo de gestión de indicadores para una empresa e venta de vehiculos," Scientia et Technica Año XIII, no 37, pp. 383-388, 2017.

[6] V.A. Arias-Vanegas, J.C. Contreras-Velasquez, V. Bermúdez, C. Garicano, M.E. Graterol-Rivas, M.E. Riaño-Garzon, J.G. Chacón-Rangel, J.C. Acevedo, O.V. Rodríguez, S.Y. Wilches, M.A. Vera, L. Portocarrero, C. Ramirez, M. Arellano-Rodríguez y O.M. Duque, "Innovación frugal: un nuevo paradigma de desarrollo sostenible en la base de la pirámide," de La base de la pirámide y la innovación frugal en América Latina, U. d. Zulia, Ed., 2017, pp. 393-408.

[7] Fundacion V\&C, "Manual de Calidad," Cúcuta, 2019.

[8] W.E. Deming, La nueva economia para la industria, el gobierno y la educacion, Diaz de santos, 1994, pp. 77-78.

[9] Ministerio de fomento, "La gestión por procesos", 2005. [En línea]. Disponible en: https://www.fomento.es/NR/rdonlyres/9541ACDE-55BF4F01-B8FA-

03269D1ED94D/19421/CaptuloIVPrincipiosdelagestindelaCali dad.pdf.

[10] O. Valle y O. Rivera, "Monitoreo e Indicadores," IDIE Guatemala educación inicial y derechos de la infancia. Sevilla, 2008.

[11] J.D. Hernandez-Bustamante, "Diseño de un sistema de indicadores de gestión para el área de ingenieria, de una empresa de servicios IPC," tesis Especialización, Área de Ciencias Adm. y Gest. UCAB, Caracas, 2006.

[12] EAE Business School, "Indicadores de gestión: la importancia de contar con ellos", 2017. [En línea]. Disponible en: https://retos-operaciones-logistica.eae.es/indicadores-degestion-la-importancia-de-contar-con-ellos/.

[13] J.L. Cardon-Olaya, A. Martinez-Carvajal, M.S. VelásquezRestrepo y Y.M. López-Fernández, "Análisis de indicadores financieros del sector manufacturero del cuero y marroquinería: un estudio sobre las empresas colombianas," Informador técnico, vol. 79, nº 2, pp. 156-168, 2015.

[14] Departamento Administrativo de la Función Pública, "Guía para la construcción de indicadores de gestión," Bogota, 2012.

[15] J.C. Bonnefoy, "Los indicares de evaluación del desempeño: una herramienta para la getión por los resultados en América Latina", 2014.

[16] R.D. Rincón, "Los Indicadores de Gestion Organizacional: Una Guia Para su Definición", Revista Universidad EAFIT, vol. 17, pp. 44-59, 1998.

[17] A. Salgueiro-Anabitarte, "de Inidcadores de Gestion y Cuadro de Mando", Días de Santos, 2001, pp. 5-6.

[18] Departamento Administrativo de la Función Pública, "Guia para la construccion de indicadores de gestión,", 2012. [En línea]. Disponible

en: http://observatoriocultural.udgvirtual.udg.mx/repositorio/bitstre am/handle/123456789/358/GuiaIndicadoresGestion.pdf?sequen ce $=1$ \&isAllowed $=y$.

[19] A.C. Gordillo-Florez, "Diseño de un sistema de gestión por procesos monitoreado por indicadores de gestión para la empresa ANDINAMEDICAL S.A. ubicada en la ciudad de Quito," tesis de grado, Facultad de Ciencias Adm. y Cont. PUCE. Ecuador, 2012.

[20] Departamento Administrativo Nacional de Estadistica, "DANE información para todos," [En línea]. Disponible en: https://www.dane.gov.co/files/planificacion/fortalecimiento/cu adernillo/Linea_base_indicadores.pdf.

[21] C.A. Solano-Cisneros, "Los Indicadores de Gestión como base de la medición de desempeño y la toma de decisiones," tesis Maestría. Área de Gest. UASB. Ecuador, 2010.

[22] J.C. Bonnefoy y M. Armijo, "Indicadores de Desempeño en el Sector Público", Santiago de Chile: ILPES, 2005.

[23] L.H. Francisco, Evaluación e Indicadores de Desempeño de Programas Publicos", 2015. [En línea]. Disponible en: http://data.evalua.cdmx.gob.mx/docs/gral/taller2015/M01EID.p df.

[24] J.M.G. Solá, "Indicadores de Gestion para las Entidades Publicas", 2013. [En línea]. Disponible en: https://pdfs.semanticscholar.org/1947/5a50f6b126fe79dd6b824 db4e54d0b71d2d8.pdf.

[25] J. Gimeno-Parello, "Calidad Bibliotecaria: Los Indicadores Como Herramientas De Medicion," InfoConexión, p. 8, 211.

[26] Ministerio de Educación Nacional, "Indicadores", 2014. [En línea]. Disponible en: http://www.modernizacionsecretarias.gov.co/index.php/tiposde-indicadores.

[27] C.A. Mejía, "Indicadores de Efectividad y Eficacia", 1998. [En línea]. Disponible en: http://www.planning.com.co/bd/valor_agregado/Octubre1998.p df.

[28] M.G. Veritier, "Factibilidad de Diseño e Implantacion de un Cuadro de Mando Integral en una Sociedad del Estado," tesis Maestría, Ciencia de la Adm. UCC. Argentina, Cordoba, 2008.

[29] R. Hernández-Sampieri, C. Fernández-Collado y M.d.P. Baptista-Lucio, "Metodología de la investigación", Mexico: McGraw-Hill, 2010.

[30] T. Ozten y C. Manterola, "Tecnicas de muestreo sobre una poblacion a estudio," Int. J. Morphol, vol. 35, n 1, pp. 227-232, 2017.

[31] J.F. Vera-Rebollo, C. Juarez-Sánchez-Rubio, A. Ramón-Morte, F.J. Torres-Alfosea, M.d.R. Navalón-García, M.d.1.p. SuchCliment, C. Baños-Castiñeira, J.E. Martinez-Pérez, J.A. IvarsBaidal, A. Triviño-Pérez, N.E. López-Hernández, J. LatorreTorrent, A.M. García-Lorca , P. Pumares-Fernandez, A. TolónBecerra, P. Rivas-Salvador , R. Lois-González, X.M. SantosSolla , P. Alonso-Logroño, C. Patiño-Romaría, E.D. GarcíaMartínez y V. Bote-Gómez, "Planificación y gestión sostenible del turismo. Propuesta y aplicación de un sistema de información turística," proyecto METASIG, CICYT. UniAlicante, España, 2001.

[32] M. Adriana, "Procedimiento de estrategia de Proyecto", 2018.

[33] I. Kaoru, "Introduction to Quality Control", First ed., Springer, Ed., USA, 2012, p. 460.

[34] H. Gutiérrez-Pulido, "de Calidad total y productividad", McGRAW-HILL, 2010, pp. 191-204.

[35] J. Castillo-Gallo y R. Vicencio-Segovia, "Propuesta de mejora 
del sistema de medición de indicadores de gestión de la calidad de la empresa ByLL SRL contratistas generales certificada bajo la Norma ISO 9001 - 2008," tesis Maestría, Direc. de la Const. UPC, Perú, 2016.

[36] M.Á. Carmona-Calvo, "Diseño de un cuadro de indicadores para la elaboración de la memoria de sostenibilidad de una organización de servicios," proyecto MPIGMA, EOI, Sevilla, 2012.

[37] C.A. Pérez-Tristancho y F. Vera-Méndez, "Fundamentos para la administración energética en la industria Colombiana a través de indicadores de," Scientia Et Technica, vol. 17, n 50, pp. 58-67, 2012.

[38] A. Russo, I. Guerreiro y O. Diaz, "Metodología para el diseño y análisis de indicadores de gestión," XLI AATN, Argentina, 2014.

[39] C. Alfaro y J. Gómez, "Un sistema de indicadores para la medición, evaluación, innovación y participación oriendado a la administracion pública," Methados.revista de ciencias sociales, vol. 4, n 2, pp. 274-290, 2016.

[40] C.A. Pérez-Cupa, A.N. Puerto-Romero y L.D. SerranoHernández, "Propuesta para estructurar un sistema de gestión de calidad basado en la norma ISO 9001:2015, en la empresa Asisderma Clínica de la piel," tesis Especialización, Facultad de Ingenierías, Uni. Agustiniana. Bogotá D.C, 2020.

[41] L.H. Francisco, "Evaluación e Indicadores de Desempeño de Programas Publicos,". [En línea]. Disponible en: http://data.evalua.cdmx.gob.mx/docs/gral/taller2015/M01EID.p df. 